\title{
The genome sequence of the hazel leaf-roller, Apoderus coryli
}

\section{(Linnaeus, 1758) [version 1; peer review: 1 approved, 1}

\section{approved with reservations]}

\author{
Liam M. Crowley (iD), \\ University of Oxford and Wytham Woods Genome Acquisition Lab, \\ Darwin Tree of Life Barcoding collective, \\ Wellcome Sanger Institute Tree of Life programme, \\ Wellcome Sanger Institute Scientific Operations: DNA Pipelines collective, \\ Tree of Life Core Informatics collective, Darwin Tree of Life Consortium \\ ${ }^{1}$ Department of Zoology, University of Oxford, Oxford, UK
}

V1 First published: 18 Nov 2021, 6:315

https://doi.org/10.12688/wellcomeopenres.17380.1

Latest published: 18 Nov 2021, 6:315

https://doi.org/10.12688/wellcomeopenres.17380.1

\section{Abstract}

We present a genome assembly from an individual male Apoderus coryli (the hazel leaf roller; Arthropoda; Insecta; Coleoptera; Attelabidae). The genome sequence is 428 megabases in span. The majority (98.90\%) of the assembly is scaffolded into 20 chromosomal pseudomolecules, with the $X$ and $Y$ sex chromosomes assembled.

Keywords

Apoderus coryli, hazel leaf roller, genome sequence, chromosomal, Coleoptera

This article is included in the Tree of Life gateway.

\section{Open Peer Review \\ Approval Status ? \\ 1 \\ 2 \\ version 1

v
view \\ 1. Khaled Amiri ID, United Arab Emirates University, Al Ain, United Arab Emirates \\ 2. Ardha Apriyanto ID, PT. Astra Agro Lestari \\ Tbk, Jakarta Timur, Indonesia \\ Any reports and responses or comments on the article can be found at the end of the article.}


Corresponding author: Darwin Tree of Life Consortium (mark.blaxter@sanger.ac.uk)

Author roles: Crowley LM: Investigation, Resources, Writing - Original Draft Preparation, Writing - Review \& Editing;

Competing interests: No competing interests were disclosed.

Grant information: This work was supported by the Wellcome Trust through core funding to the Wellcome Sanger Institute (206194) and the Darwin Tree of Life Discretionary Award (218328).

The funders had no role in study design, data collection and analysis, decision to publish, or preparation of the manuscript.

Copyright: (c) 2021 Crowley LM et al. This is an open access article distributed under the terms of the Creative Commons Attribution License, which permits unrestricted use, distribution, and reproduction in any medium, provided the original work is properly cited.

How to cite this article: Crowley LM, University of Oxford and Wytham Woods Genome Acquisition Lab, Darwin Tree of Life Barcoding collective et al. The genome sequence of the hazel leaf-roller, Apoderus coryli (Linnaeus, 1758) [version 1; peer review: 1 approved, 1 approved with reservations] Wellcome Open Research 2021, 6:315 https://doi.org/10.12688/wellcomeopenres.17380.1

First published: 18 Nov 2021, 6:315 https://doi.org/10.12688/wellcomeopenres.17380.1 


\section{Species taxonomy}

Eukaryota; Metazoa; Ecdysozoa; Arthropoda; Hexapoda; Insecta; Pterygota; Neoptera; Endopterygota; Coleoptera; Polyphaga; Cucujiformia; Attelabidae; Apoderinae; Apoderus; Apoderus coryli (Linnaues, 1758) (NCBI:txid201766).

\section{Background}

The hazel leaf-roller, Apoderus coryli, is a distinctive leafrolling weevil which is widespread and locally common across Europe and western Asia. In the UK, it is common in deciduous woodland across England and Wales, with a few records from southern Scotland. The main host plant is common hazel, Corylus avellana, although it is also known to infrequently feed on alder, birch, hornbeam and beech. It may be considered a minor pest of hazelnut cultivation (Gantner, 2000). Adults are $6-8 \mathrm{~mm}$ with red elytra, pronotum and bases of the femora. The head, underside and remainder of the legs are black. It has a distinctive elongate head and 'neck'. Adults are active from May, feeding on hazel leaves in which they leave characteristic round holes. Mating and oviposition occurs in early summer. Females lay a single bright orange egg around $1 \mathrm{~mm}$ in size on a leaf before cutting a line across the leaf with their mandibles and rolling it into a cylinder around the egg. The leaf rolling process may take 60-90 minutes and a single female may repeat this for up to 30 eggs across several days (Urban, 2014). Larvae develop and pupate within these leaf rolls. Second generation adults are active in late summer, the larvae of which overwinter within leaf rolls that drop to the ground and pupate in the spring. A previous study has shown that this species has 13 autosomal bivalents, that sex chromosomes exhibit a parachute association and that this species possesses a small amount of heterochromatin (Rozek et al., 2004).

\section{Genome sequence report}

The genome was sequenced from one male A. coryli (Figure 1) collected from Wytham Woods, Oxfordshire (biological vicecounty: Berkshire), UK (latitude 51.770, longitude -1.339). A total of 59-fold coverage in Pacific Biosciences single-molecule long reads and 93-fold coverage in 10X Genomics read clouds were generated. Primary assembly contigs were scaffolded with chromosome conformation $\mathrm{Hi}-\mathrm{C}$ data. Manual assembly curation corrected 57 missing/misjoins and removed 7 haplotypic duplications, reducing the assembly length by $0.41 \%$ and the scaffold number by $24.71 \%$, and increasing the scaffold N50 by $24.75 \%$.

The final assembly has a total length of $428 \mathrm{Mb}$ in 128 sequence scaffolds with a scaffold N50 of $23.7 \mathrm{Mb}$ (Table 1). The majority, $98.90 \%$, of the assembly sequence was assigned to 20 chromosomal-level scaffolds, representing 18 autosomes (numbered by sequence length), and the $\mathrm{X}$ and $\mathrm{Y}$ sex chromosome (Figure 2-Figure 5; Table 2). The assembly has a BUSCO v5.1.2 (Simão et al., 2015) completeness of $99.3 \%$ (single $97.7 \%$, duplicated $1.6 \%$ ) using the endopterygota_odb10 reference set. While not fully phased, the assembly deposited is of one haplotype. Contigs corresponding to the second haplotype have also been deposited.

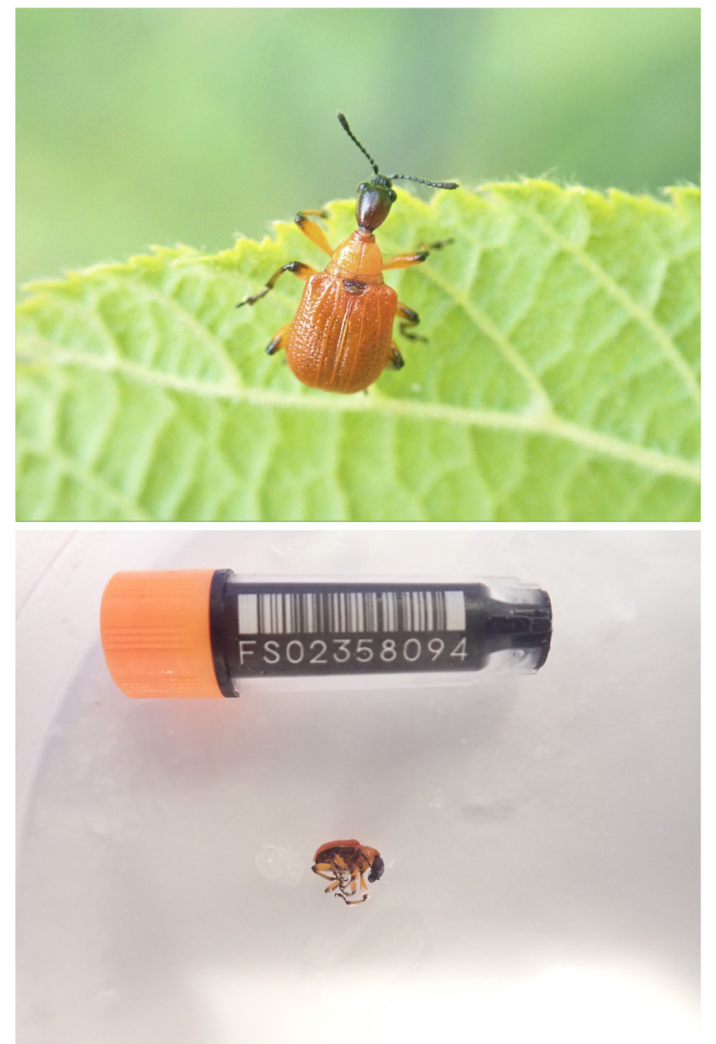

Figure 1. Images of the Apoderus colyri specimen, icApoCory1, used for genome sequencing. Top, the specimen prior to collection; bottom, the specimen during preservation and processing, shown with a $43.9 \mathrm{~mm}$ FluidX sample tube.

\section{Methods}

Sample acquisition and DNA extraction

A single male $A$. coryli was collected from Wytham Woods, Oxfordshire (biological vice-county: Berkshire), UK (latitude 51.770, longitude -1.339) by Liam Crowley, University of Oxford, using a pooter. The sample was snap-frozen on dry ice and stored using a CoolRack.

DNA was extracted at the Tree of Life laboratory, Wellcome Sanger Institute. The icApoCory 1 sample was weighed and dissected on dry ice with tissue set aside for $\mathrm{Hi}-\mathrm{C}$ sequencing. Tissue from the whole organism was cryogenically disrupted to a fine powder using a Covaris cryoPREP Automated Dry Pulveriser, receiving multiple impacts. Fragment size analysis of 0.01-0.5 ng of DNA was then performed using an Agilent FemtoPulse. High molecular weight (HMW) DNA was extracted using the Qiagen MagAttract HMW DNA extraction kit. Low molecular weight DNA was removed from a 200-ng aliquot of extracted DNA using $0.8 \mathrm{X}$ AMpure XP purification kit prior to $10 \mathrm{X}$ Chromium sequencing; a minimum of $50 \mathrm{ng}$ DNA was submitted for $10 \mathrm{X}$ sequencing. HMW DNA was sheared into an average fragment size between $12-20 \mathrm{~kb}$ in a Megaruptor 3 system with speed setting 30. Sheared DNA was purified 


\section{Table 1. Genome data for Apoderus coryli, icApoCory1.1.}

\begin{tabular}{|c|c|}
\hline \multicolumn{2}{|l|}{ Project accession data } \\
\hline Assembly identifier & icApoCory1.1 \\
\hline Species & Apoderus coryli \\
\hline Specimen & icApoCory1 \\
\hline NCBI taxonomy ID & NCBI:txid201766 \\
\hline BioProject & PRJEB45119 \\
\hline BioSample ID & SAMEA7520690 \\
\hline Isolate information & Male, whole organism \\
\hline \multicolumn{2}{|l|}{ Raw data accessions } \\
\hline PacificBiosciences SEQUEL II & ERR6412370 \\
\hline 10X Genomics Illumina & ERR6054772-ERR6054775 \\
\hline Hi-C Illumina & ERR6054771 \\
\hline \multicolumn{2}{|l|}{ Genome assembly } \\
\hline Assembly accession & GCA_911728435.1 \\
\hline Accession of alternate haplotype & GCA_911728465.1 \\
\hline Span (Mb) & 428 \\
\hline Number of contigs & 203 \\
\hline Contig N50 length (Mb) & 15.0 \\
\hline Number of scaffolds & 128 \\
\hline Scaffold N50 length (Mb) & 23.7 \\
\hline Longest scaffold (Mb) & 46.0 \\
\hline BUSCO* genome score & $\begin{array}{l}\text { C:99.3\%[S:97.7\%,D:1.6\%], } \\
\text { F:0.4\%,M:0.3\%,n:2124 }\end{array}$ \\
\hline \multicolumn{2}{|c|}{$\begin{array}{l}\text { *BUSCO scores based on the endopterygota_odb10 BUSCO set using } \\
\text { v5.1.2. C= complete [S= single copy, D=duplicated], F=fragmented, } \\
\text { M=missing, n=number of orthologues in comparison. A full set of } \\
\text { BUSCO scores is available at https://blobtoolkit.genomehubs.org/view/ } \\
\text { icApoCory1.1/dataset/CAJVRQ01/busco. }\end{array}$} \\
\hline
\end{tabular}

by solid-phase reversible immobilisation using AMPure PB beads with a $1.8 \mathrm{X}$ ratio of beads to sample to remove the shorter fragments and concentrate the DNA sample. The concentration of the sheared and purified DNA was assessed using a Nanodrop spectrophotometer and Qubit Fluorometer and Qubit dsDNA High Sensitivity Assay kit. Fragment size distribution was evaluated by running the sample on the FemtoPulse system.

\section{Sequencing}

Pacific Biosciences HiFi circular consensus and 10X Genomics read cloud DNA sequencing libraries were constructed according to the manufacturers' instructions. Sequencing was performed by the Scientific Operations core at the Wellcome Sanger Institute on Pacific Biosciences SEQUEL II and Illumina HiSeq $\mathrm{X}$ instruments. Hi-C data were generated from further whole organism tissue using the Arima v2 $\mathrm{Hi}-\mathrm{C}$ kit and sequenced on a HiSeq X instrument.

\section{Genome assembly}

Assembly was carried out with Hifiasm (Cheng et al., 2021); haplotypic duplication was identified and removed with purge_ dups (Guan et al., 2020) with the -e flag. One round of polishing was performed by aligning $10 \mathrm{X}$ Genomics read data to the assembly with longranger align, calling variants with 
Scaffold statistics

BUSCO endopterygota_odb10 (2124)

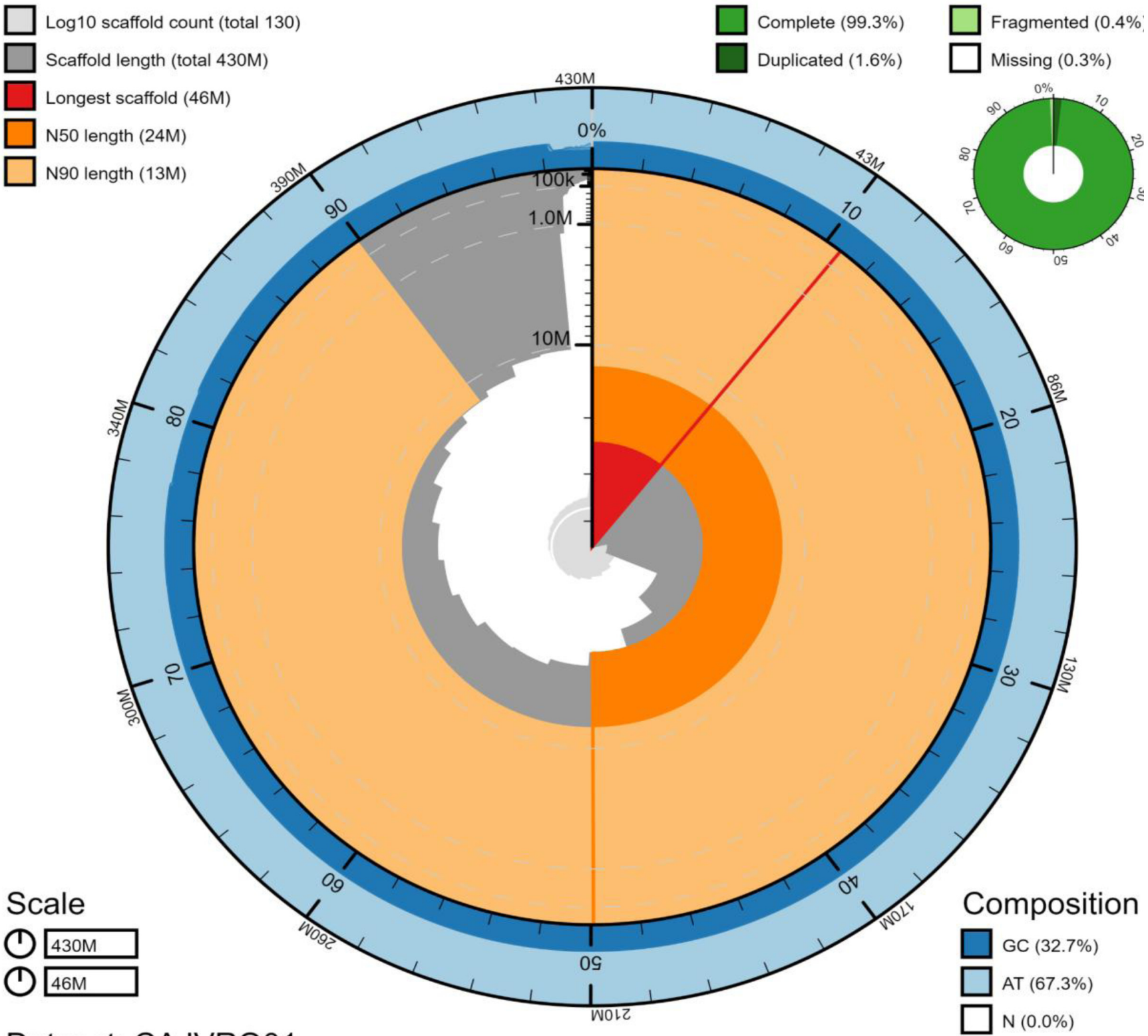

Dataset: CAJVRQ01

Figure 2. Genome assembly of Apoderus coryli, icApoCory1.1: metrics. The BlobToolKit Snailplot shows N50 metrics and BUSCO gene completeness. The main plot is divided into 1,000 size-ordered bins around the circumference with each bin representing $0.1 \%$ of the $428,077,793 \mathrm{bp}$ assembly. The distribution of scaffold lengths is shown in dark grey with the plot radius scaled to the longest scaffold present in the assembly $(45,983,548 \mathrm{bp}$, shown in red). Orange and pale-orange arcs show the N50 and N90 scaffold lengths $(23,694,900$ and $12,555,284 \mathrm{bp})$, respectively. The pale grey spiral shows the cumulative scaffold count on a log scale with white scale lines showing successive orders of magnitude. The blue and pale-blue area around the outside of the plot shows the distribution of GC, AT and N percentages in the same bins as the inner plot. A summary of complete, fragmented, duplicated and missing BUSCO genes in the endopterygota_odb10 set is shown in the top right. An interactive version of this figure is available at https:// blobtoolkit.genomehubs.org/view/icApoCory1.1/dataset/CAJVRQ01/snail.

freebayes (Garrison \& Marth, 2012). The assembly was then scaffolded with Hi-C data (Rao et al., 2014) using SALSA2
(Ghurye et al., 2019). The assembly was checked for contamination and corrected using the gEVAL system (Chow et al., 2016) 


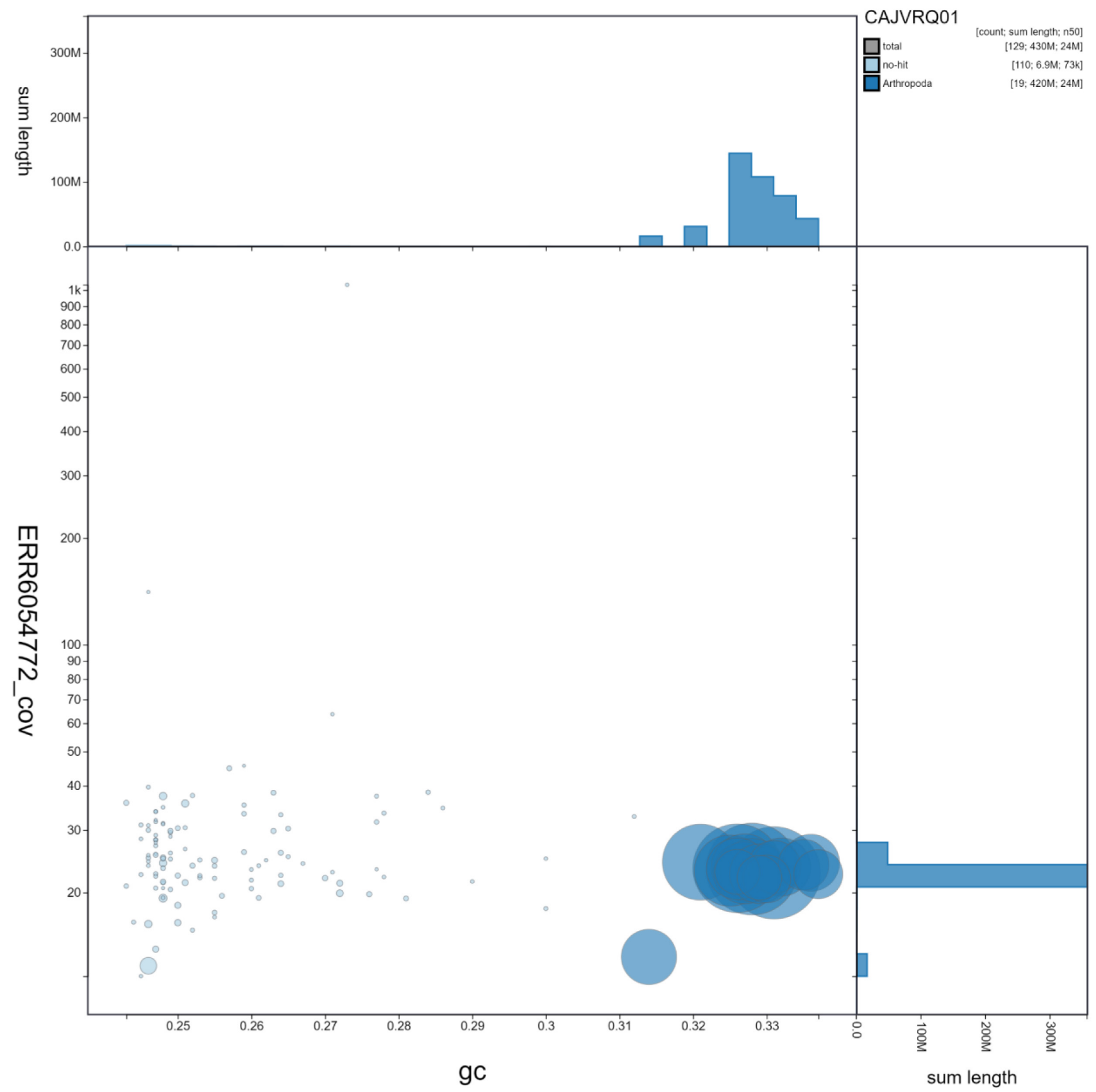

Figure 3. Genome assembly of Apoderus coryli, icApoCory1.1: GC coverage. BlobToolKit GC-coverage plot. Scaffolds are coloured by phylum. Circles are sized in proportion to scaffold length. Histograms show the distribution of scaffold length sum along each axis. An interactive version of this figure is available at https://blobtoolkit.genomehubs.org/view/icApoCory1.1/dataset/CAJVRQ01/blob.

as described previously (Howe et al., 2021). Manual curation was performed using gEVAL, HiGlass (Kerpedjiev et al., 2018) and Pretext. The mitochondrial genome was assembled using MitoHiFi (Uliano-Silva et al., 2021) and annotated with
MitoFinder (Allio et al., 2020). The genome was analysed and BUSCO scores generated within the BlobToolKit environment (Challis et al., 2020). Table 3 contains a list of all software tool versions used, where appropriate. 


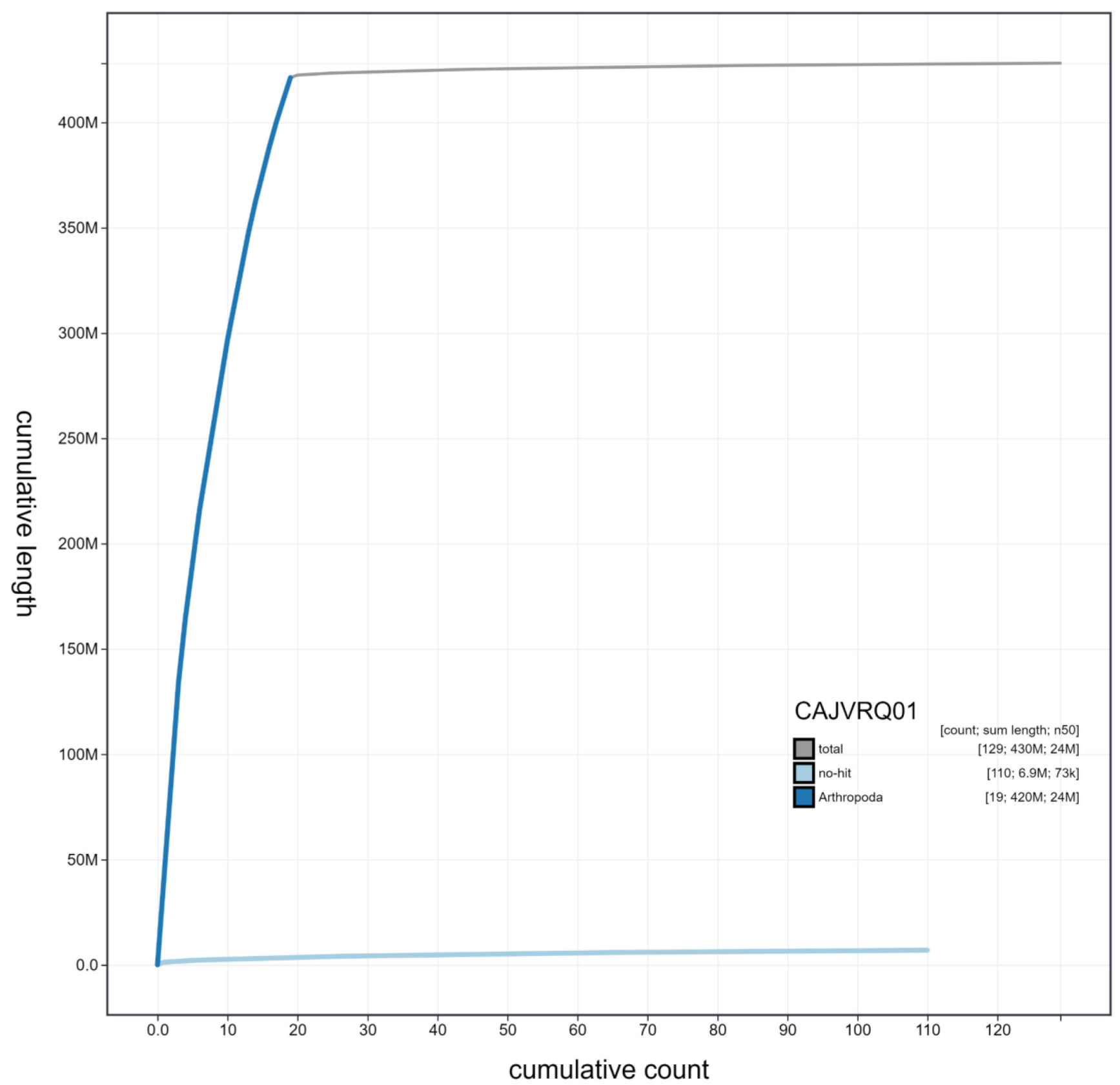

Figure 4. Genome assembly of Apoderus coryli, icApoCory1.1: cumulative sequence. BlobToolKit cumulative sequence plot. The grey line shows cumulative length for all scaffolds. Coloured lines show cumulative lengths of scaffolds assigned to each phylum using the buscogenes taxrule. An interactive version of this figure is available at https://blobtoolkit.genomehubs.org/view/icRhaFulv1.1/dataset/ CAJPIC01.1/cumulative.

\section{Ethics/compliance issues}

The materials that have contributed to this genome note have been supplied by a Darwin Tree of Life Partner. The submission of materials by a Darwin Tree of Life Partner is subject to the
Darwin Tree of Life Project Sampling Code of Practice. By agreeing with and signing up to the Sampling Code of Practice, the Darwin Tree of Life Partner agrees they will meet the legal and ethical requirements and standards set out within this 


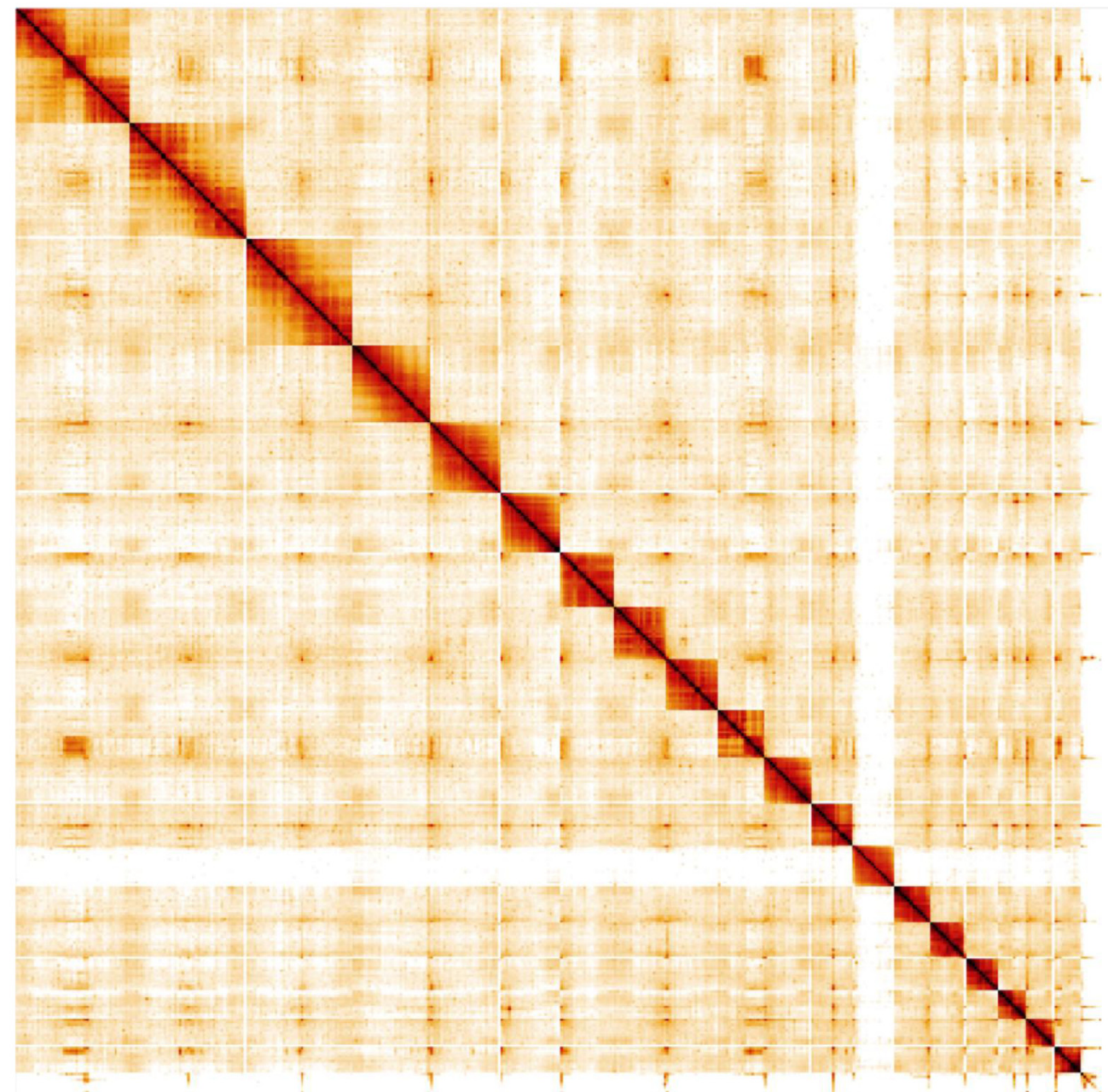

Figure 5. Genome assembly of Apoderus coryli, icApoCory1.1: Hi-C contact map. Hi-C contact map of the icApoCory1.1 assembly, visualised in HiGlass.

\begin{tabular}{|c|c|c|c|}
\hline INSDC accession & Chromosome & Size (Mb) & GC\% \\
\hline OU452198.1 & 1 & 45.98 & 33.1 \\
\hline OU452199.1 & 2 & 45.50 & 32.8 \\
\hline OU452200.1 & 3 & 42.38 & 32.6 \\
\hline OU452201.1 & 4 & 30.93 & 32.1 \\
\hline OU452202.1 & 5 & 27.11 & 32.5 \\
\hline OU452203.1 & 6 & 23.69 & 32.7 \\
\hline OU452204.1 & 7 & 21.42 & 32.8 \\
\hline OU452205.1 & 8 & 20.46 & 32.7 \\
\hline OU452206.1 & 9 & 20.22 & 32.6 \\
\hline OU452207.1 & 10 & 18.89 & 32.9 \\
\hline
\end{tabular}

\begin{tabular}{|c|c|c|c|}
\hline INSDC accession & Chromosome & Size (Mb) & $\mathbf{G C}$ \\
\hline OU452208.1 & 11 & 17.89 & 33.2 \\
\hline OU452209.1 & 12 & 17.13 & 33.6 \\
\hline OU452211.1 & 13 & 14.69 & 33.1 \\
\hline OU452212.1 & 14 & 13.58 & 33.5 \\
\hline OU452213.1 & 15 & 12.56 & 33.7 \\
\hline OU452214.1 & 16 & 11.87 & 33 \\
\hline OU452215.1 & 17 & 10.51 & 32.6 \\
\hline OU452216.1 & 18 & 10.30 & 32.9 \\
\hline OU452210.1 & X & 16.03 & 31.4 \\
\hline OU452217.1 & Y & 1.26 & 24.7 \\
\hline OU452218.1 & MT & 0.02 & 27.3 \\
\hline- & Unplaced & 5.65 & 25.5 \\
\hline
\end{tabular}




\section{Table 3. Software tools used.}

\begin{tabular}{|l|l|l|}
\hline Software tool & Version & Source \\
\hline Hifiasm & 0.12 & Cheng et al., 2021 \\
\hline purge_dups & 1.2 .3 & Guan et al., 2020 \\
\hline SALSA2 & 2.2 & Ghurye et al., 2019 \\
\hline Iongranger align & 2.2 .2 & $\begin{array}{l}\text { https://support.10xgenomics.com/genome-exome/ } \\
\text { software/pipelines/latest/advanced/other-pipelines }\end{array}$ \\
\hline freebayes & $1.3 .1-17$-gaa2ace8 & Garrison \& Marth, 2012 \\
\hline MitoHiFi & 2.1 & Uliano-Silva et al., 2021 \\
\hline gEVAL & N/A & Chow et al., 2016 \\
\hline HiGlass & 1.11 .6 & Kerpedjiev et al., 2018 \\
\hline PretextView & $0.1 . x$ & https://github.com/wtsi-hpag/PretextView \\
\hline BlobToolKit & 2.6 .2 & Challis et al., 2020 \\
\hline
\end{tabular}

document in respect of all samples acquired for, and supplied to, the Darwin Tree of Life Project. Each transfer of samples is further undertaken according to a Research Collaboration Agreement or Material Transfer Agreement entered into by the Darwin Tree of Life Partner, Genome Research Limited (operating as the Wellcome Sanger Institute), and in some circumstances other Darwin Tree of Life collaborators.

\section{Data availability}

European Nucleotide Archive: Apoderus coryli (hazel leaf-roller). Accession number PRJEB45119; https://identifiers.org/ena.embl/ PRJEB45119.

The genome sequence is released openly for reuse. The A. coryli genome sequencing initiative is part of the Darwin Tree of Life (DToL) project. All raw sequence data and the assembly have been deposited in INSDC databases. The genome will be annotated and presented through the Ensembl pipeline at the European Bioinformatics Institute. Raw data and assembly accession identifiers are reported in Table 1.

\section{Author information}

Members of the University of Oxford and Wytham Woods Genome Acquisition Lab are listed here: https://doi.org/10.5281/ zenodo.4789929.

Members of the Darwin Tree of Life Barcoding collective are listed here: https://doi.org/10.5281/zenodo.4893704.

Members of the Wellcome Sanger Institute Tree of Life programme collective are listed here: https://doi.org/10.5281/ zenodo.5377053.

Members of Wellcome Sanger Institute Scientific Operations: DNA Pipelines collective are listed here: https://doi.org/10.5281/ zenodo.4790456.

Members of the Tree of Life Core Informatics collective are listed here: https://doi.org/10.5281/zenodo.5013542.

Members of the Darwin Tree of Life Consortium are listed here: https://doi.org/10.5281/zenodo.4783559.
Allio R, Schomaker-Bastos A, Romiguier J, et al.: MitoFinder: Efficient automated large-scale extraction of mitogenomic data in target enrichment phylogenomics. Mol Ecol Resour. 2020; 20(4): 892-905. PubMed Abstract | Publisher Full Text | Free Full Text

Challis R, Richards E, Rajan J, et al.: BlobToolKit--Interactive Quality Assessment of Genome Assemblies. G3 (Bethesda). 2020; 10(4): 1361-74. PubMed Abstract | Publisher Full Text | Free Full Text

Cheng $\mathrm{H}$, Concepcion GT, Feng $\mathrm{X}$, et al:: Haplotype-Resolved de Novo Assembly Using Phased Assembly Graphs with Hifiasm. Nat Methods. 2021; 18(2): 170-75.

PubMed Abstract | Publisher Full Text | Free Full Text

Chow W, Brugger K, Caccamo M, et al.: gEVAL - a Web-Based Browser for
Evaluating Genome Assemblies. Bioinformatics. 2016; 32(16): 2508-10. PubMed Abstract | Publisher Full Text | Free Full Text

Gantner M: Occurrence of Hazelnut Pests in Southeastern Poland. In V International Congress on Hazelnut. 2000; 556: 469-78. Publisher Full Text

Garrison E, Marth G: Haplotype-Based Variant Detection from Short-Read Sequencing. arXiv 1207.3907, 2012

Reference Source

Ghurye J, Rhie A, Walenz BP, et al.: Integrating Hi-C Links with Assembly Graphs for Chromosome-Scale Assembly. PLoS Comput Biol. 2019; 15(8): e1007273.

PubMed Abstract | Publisher Full Text | Free Full Text 
Guan D, McCarthy SA, Wood J, et al.: Identifying and Removing Haplotypic Duplication in Primary Genome Assemblies. Bioinformatics. 2020; 36(9): 2896-2898.

PubMed Abstract | Publisher Full Text | Free Full Text

Howe K, Chow W, Collins J, et al.: Significantly Improving the Quality of Genome Assemblies through Curation. Gigascience. 2021; 10(1):

giaa153.

PubMed Abstract | Publisher Full Text | Free Full Text

Kerpedjiev P, Abdennur N, Lekschas F, et al:: HiGlass: Web-Based Visual Exploration and Analysis of Genome Interaction Maps. Genome Biol. 2018; 19(1): 125

PubMed Abstract | Publisher Full Text | Free Full Text

Rao SSP, Huntley MH, Durand NC, et al:: A 3D Map of the Human Genome at Kilobase Resolution Reveals Principles of Chromatin Looping. Cell. 2014;

159(7): 1665-80.

PubMed Abstract | Publisher Full Text | Free Full Text
Rozek M, Lachowska D, Petitpierre E, et al.: C-Bands on Chromosomes of 32 Beetle Species (Coleoptera: Elateridae, Cantharidae, Oedemeridae, Cerambycidae, Anthicidae, Chrysomelidae, Attelabidae and Curculionidae). Hereditas. 2004; 140(3): 161-70.

PubMed Abstract | Publisher Full Text

Simão FA, Waterhouse RM, Ioannidis P, et al.: BUSCO: Assessing Genome Assembly and Annotation Completeness with Single-Copy Orthologs. Bioinformatics. 2015; 31(19): 3210-12.

PubMed Abstract | Publisher Full Text

Uliano-Silva M, Nunes JGF, Krasheninnikova K, et al.: marcelauliano/MitoHiFi: mitohifi_v2.0. 2021.

Publisher Full Text

Urban J: Apoderus Coryli (L.) - a Biologically Little Known Species of the Attelabidae (Coleoptera). Acta Univ Agric Silvic Mendelianae Brun. 2014; 62(5): 1141-60.

Publisher Full Text 


\section{Open Peer Review}

\section{Current Peer Review Status:}

\section{Version 1}

Reviewer Report 01 December 2022

https://doi.org/10.21956/wellcomeopenres.19217.r53152

(c) 2022 Apriyanto A. This is an open access peer review report distributed under the terms of the Creative Commons Attribution License, which permits unrestricted use, distribution, and reproduction in any medium, provided the original work is properly cited.

\section{Ardha Apriyanto}

Research and Development, PT. Astra Agro Lestari Tbk, Jakarta Timur, Indonesia

In this report, the authors present a genome sequence of Apoderus coryli (Linnaeus, 1758), the hazel leaf-roller. The sequence reported is novel and original. Furthermore, the dataset will provide a useful resource for the scientific community. This reported genome produced a highquality genome assembly that utilizes various sequencing technologies. However, this report does not address the genome sequence annotation.

Regarding the manuscript, I have some minor comments:

1. There is a duplicate sentence regarding sample collection in the Genome sequence report and Methods section. "collected from Wytham Woods, Oxfordshire (biological vice-county: Berkshire), UK (latitude 51.770, longitude -1.339)." Please rewrite or omit one of them since it is redundant.

2. Please include a bar scale in Figure 1.

3. The link in Figure 4 is wrong. It belongs to the R.fulva CAJPICO1. Please revise this.

Overall, I would endorse it for indexing with minor revisions.

Is the rationale for creating the dataset(s) clearly described?

Yes

Are the protocols appropriate and is the work technically sound?

Yes

Are sufficient details of methods and materials provided to allow replication by others? Yes

Are the datasets clearly presented in a useable and accessible format? Yes 
Competing Interests: No competing interests were disclosed.

Reviewer Expertise: Genome assembly, Omics (Genomics, Transcriptomics, Proteomics, Metabolomics), Bioinformatics

I confirm that I have read this submission and believe that I have an appropriate level of expertise to confirm that it is of an acceptable scientific standard, however I have significant reservations, as outlined above.

Reviewer Report 02 December 2021

https://doi.org/10.21956/wellcomeopenres.19217.r47132

(C) 2021 Amiri K. This is an open access peer review report distributed under the terms of the Creative Commons Attribution License, which permits unrestricted use, distribution, and reproduction in any medium, provided the original work is properly cited.

\section{Khaled Amiri}

Department of Biology, College of Science, United Arab Emirates University, Al Ain, United Arab Emirates

The Genome assembly of the hazel leaf-roller, Apoderus coryli is completely descriptive but provides a well-assembled genome with 20 chromosomal pseudomolecules. The author has arrived to this assembly using several genomic platforms. The figures present the cumulative sequence and other parameters. I would, however, briefly compare the sequence presented here with other beetles.

Is the rationale for creating the dataset(s) clearly described?

Yes

Are the protocols appropriate and is the work technically sound?

Yes

Are sufficient details of methods and materials provided to allow replication by others? Yes

Are the datasets clearly presented in a useable and accessible format? Yes

Competing Interests: No competing interests were disclosed.

Reviewer Expertise: Genome science

I confirm that I have read this submission and believe that I have an appropriate level of expertise to confirm that it is of an acceptable scientific standard. 